\title{
Minimizing Postsampling Degradation of Peptides by a Thermal Benchtop Tissue Stabilization Method
}

\author{
Lova Segerström, ${ }^{*}$ Jenny Gustavsson, ${ }^{*}$ and Ingrid Nylander
}

Enzymatic degradation is a major concern in peptide analysis. Postmortem metabolism in biological samples entails considerable risk for measurements misrepresentative of true in vivo concentrations. It is therefore vital to find reliable, reproducible, and easy-to-use procedures to inhibit enzymatic activity in fresh tissues before subjecting them to qualitative and quantitative analyses. The aim of this study was to test a benchtop thermal stabilization method to optimize measurement of endogenous opioids in brain tissue. Endogenous opioid peptides are generated from precursor proteins through multiple enzymatic steps that include conversion of one bioactive peptide to another, often with a different function. Ex vivo metabolism may, therefore, lead to erroneous functional interpretations. The efficacy of heat stabilization was systematically evaluated in a number of postmortem handling procedures. Dynorphin B (DYNB), Leu-enkephalin-Arg ${ }^{6}$ (LARG), and Met-enkephalin$\mathrm{Arg}^{6}-\mathrm{Phe}^{7}$ (MEAP) were measured by radioimmunoassay in rat hypothalamus, striatum (STR), and cingulate cortex (CCX). Also, simplified extraction protocols for stabilized tissue were tested. Stabilization affected all peptide levels to varying degrees compared to those prepared by standard dissection and tissue handling procedures. Stabilization increased DYNB in hypothalamus, but not STR or CCX, whereas LARG generally decreased. MEAP increased in hypothalamus after all stabilization procedures, whereas for STR and CCX, the effect was dependent on the time point for stabilization. The efficacy of stabilization allowed samples to be left for 2 hours in room temperature $\left(20^{\circ} \mathrm{C}\right)$ without changes in peptide levels. This study shows that conductive heat transfer is an easy-to-use and efficient procedure for the preservation of the molecular composition in biological samples. Region- and peptide-specific critical steps were identified and stabilization enabled the optimization of tissue handling and opioid peptide analysis. The result is improved diagnostic and research value of the samples with great benefits for basic research and clinical work.

\section{Introduction}

$\mathrm{P}$ OSTMORTEM METABOLISM IS A major concern in the analysis of peptides in biological samples and it is vital to find reliable, reproducible, and easy-to-use procedures to avoid this issue when handling fresh tissues. A number of enzymes participate in peptide turnover, and without resolute inactivation of these processes there is a considerable risk that measured levels are not representative of those in vivo. ${ }^{1,2}$

Decapitation followed by dissection on wet ice and freezing on dry ice is a common procedure for brain tissue sampling. Lowering the temperature decreases the enzymatic activity, although the lag between decapitation and freezing still allows considerable time for enzymatic ac- tions. Furthermore, samples are commonly thawed and/or reheated during extraction procedures, which again increases enzymatic activity. Strategies to avoid enzymatic processes include thermal inactivation, protease inhibition, acidic $\mathrm{pH}$, etc. ${ }^{3,4}$ Thermal inactivation is considered the superior strategy because no extraneous components are added to the sample and the enzymes are irreversibly denatured. Methods for thermal inactivation of biological samples are through either conductive heat transfer ${ }^{5}$ or microwave irradiation, ${ }^{6}$ both of which result in almost immediate inactivation of enzymes. Several studies have shown an increased concentration of peptides in brain samples after microwave irradiation. ${ }^{7-9}$

Endogenous opioid peptides are derived from the precursors proenkephalin, ${ }^{10}$ proopiomelanocortin, ${ }^{11}$ and prodynorphin ${ }^{12}$

Department of Pharmaceutical Biosciences, Neuropharmacology, Addiction \& Behavior, Uppsala University, Uppsala, Sweden.

*These authors contributed equally to this article.

(C) Lova Segerström et al., 2016; Published by Mary Ann Liebert, Inc. This Open Access article is distributed under the terms of the Creative Commons Attribution Noncommercial License (http://creativecommons.org/licenses/by-nc/4.0/) which permits any noncommercial use, distribution, and reproduction in any medium, provided the original author(s) and the source are credited. 
through enzymatic cleavage. ${ }^{2,13}$ One precursor generates several bioactive opioid peptides, for example, dynorphins and enkephalins from prodynorphin, and these often mediate different or even opposing physiological effects. ${ }^{14,15}$ Thus, to avoid erroneous physiological interpretations and conclusions from measured peptide levels due to poor association between measured and in vivo levels, it is of interest to minimize enzymatic activity. This was highlighted by a study comparing focused cranial in vivo microwave irradiation to conventional decapitation. Microwave irradiation led to increased peptide levels that implied cessation of degradation. ${ }^{8}$

The present study used a recently developed instrument that stabilizes tissue samples through conductive heat transfer. This method aims toward standardization of rapid and efficient enzyme inactivation. Studies validating the instrument have revealed enzymatic activity reduced to background levels after stabilization, ${ }^{4}$ increased detection of endogenous peptides and reduced postmortem degradation fragments, ${ }^{5,16}$ and sustained posttranslational protein phosphorylation despite room-temperature stor- age. ${ }^{17}$ These findings consolidate the advantages of the instrument in both basic research and the clinical setting. The current study focuses on whether this technique could optimize measurements of endogenous opioids in brain tissue. Various postmortem handling procedures were used to systematically evaluate heat stabilization of dynorphin B (DYNB), Leu-enkephalin-Arg ${ }^{6}$ (LARG), and Met-enkephalin-Arg ${ }^{6}-\mathrm{Phe}^{7}$ (MEAP) in three brain areas (hypothalamus, striatum [STR], and cingulate cortex $[\mathrm{CCX}]$ ), and second, test new protocols for peptide extraction of stabilized tissues.

\section{Materials and Methods}

\section{Animals}

Male adolescent (200 g, 7 weeks) Wistar rats (RccHan: WIST, Harlan Laboratories) were housed three per cage with food and water ad libitum in temperature- and humiditycontrolled rooms on a reversed 12-h light-12-h dark cycle
A

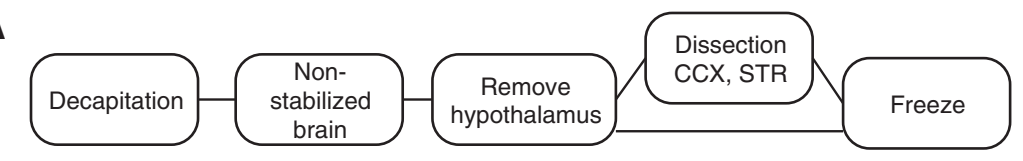

B

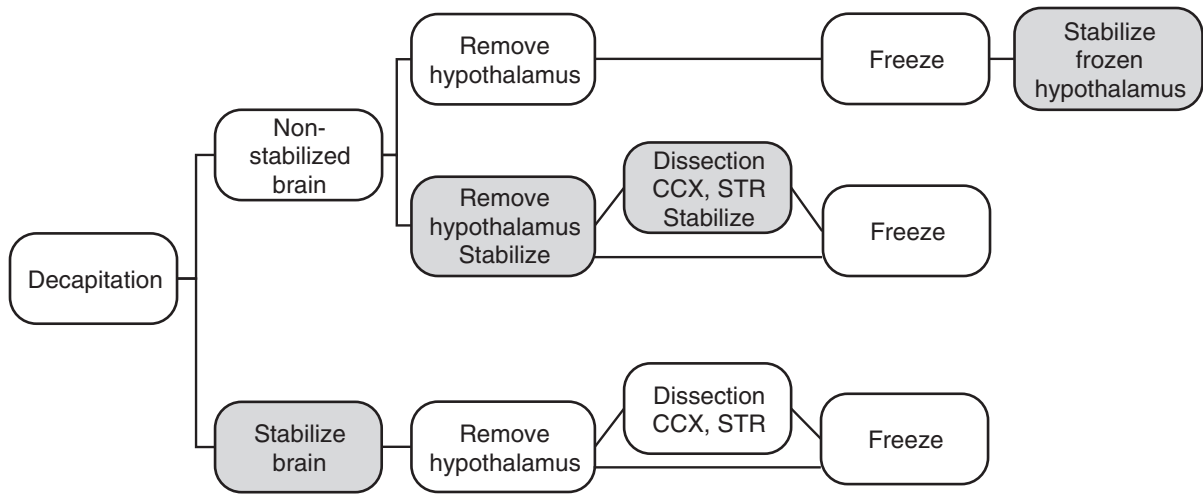

C

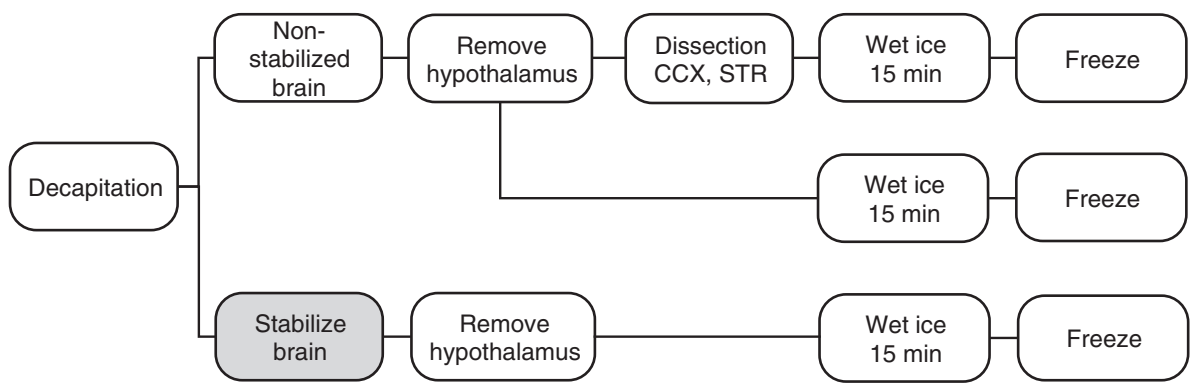

D

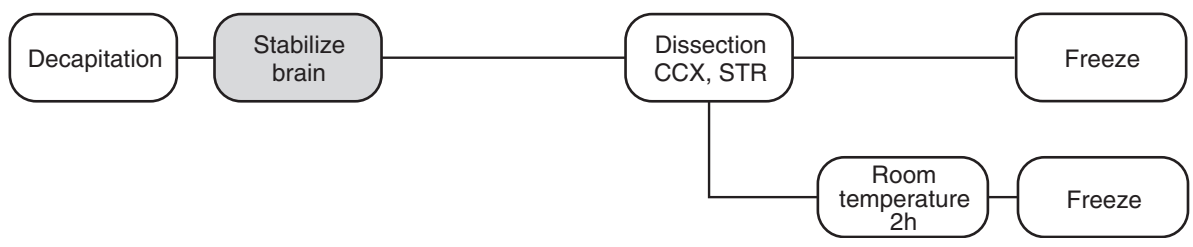

FIG. 1. Experimental outline of Part I. (A) Standard procedure for dissection of the brain and tissue collection; (B) the three stabilization procedures (see text); (C) test procedures used to examine changes in peptide levels in samples placed on wet ice for 15 minutes; (D) test procedure to examine the efficiency of the stabilization procedure. CCX, cingulate cortex; STR, striatum. 
FIG. 2. Experimental outline of Part II. Hypothalamus and the forebrain region from stabilized brain were pooled as homogenous samples, then aliquots were subjected to five different extraction procedures. HAc, acetic acid; $\mathrm{MeOH}$, methanol; RIA, radioimmunoassay; W, water.

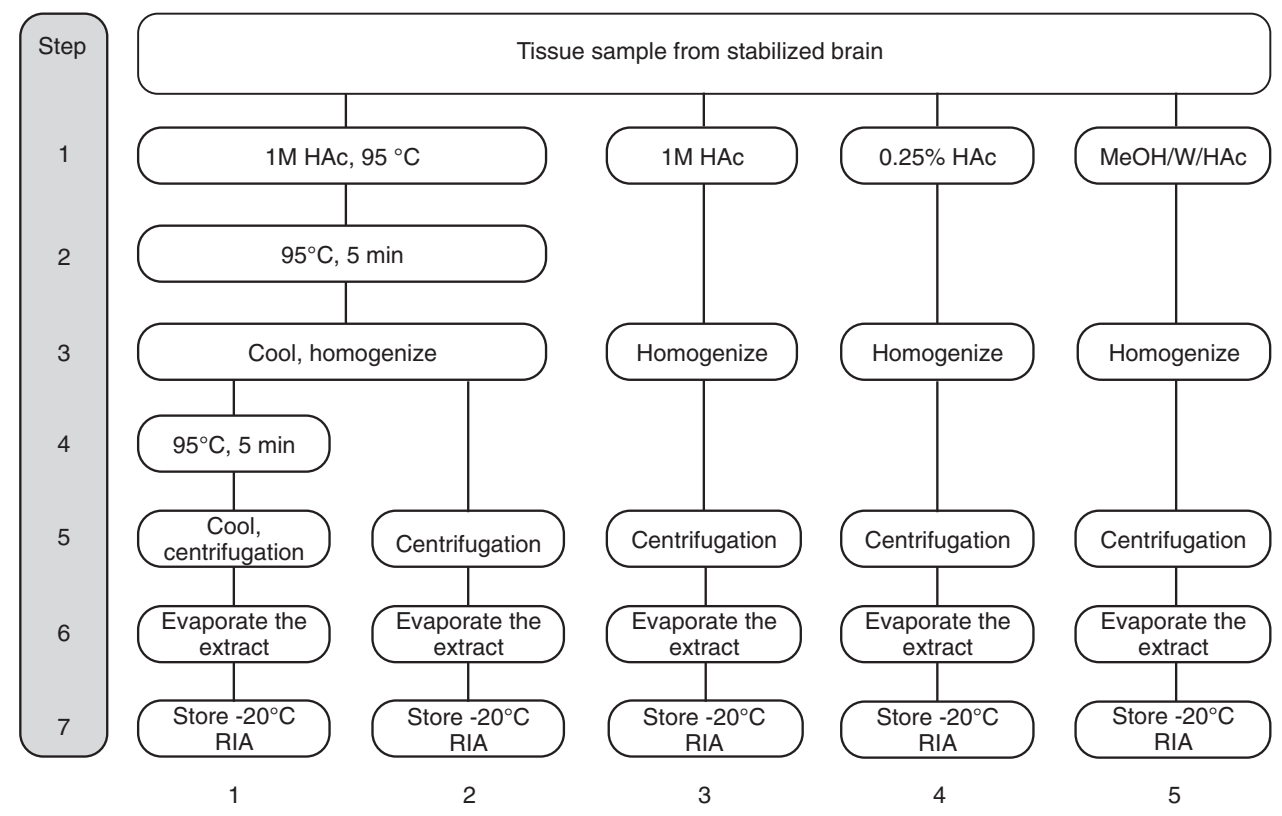

(lights off at 6:00 a.m.). They were acclimatized 1 week before the experiments, which were approved by the regional ethics committee and in accordance with the Swedish Animal Protection Legislation.

\section{Choice of brain areas}

Criteria for the choice of brain area structures were that they were easily identifiable, well-described regarding opioid content, and could be dissected out at different time points. In the first part of the study (I), three structures were examined, that is, hypothalamus (rapidly collected and minimally exposed to postmortem change), the STR and the CCX (containing high and low dynorphin levels, respectively, and dissected from a single brain section to minimize time differences). In the second part (II), pooled homogenates of hypothalamus or the forebrain region were used to test different extraction protocols.

\section{Dissection}

After decapitation, the hypothalamus was removed and the brain, placed in an ice-chilled metal brain matrix (ASI Instruments, Inc.), was sectioned in $1 \mathrm{~mm}$ coronal sections front to back. STR and CCX were manually dissected from sections with guidance of a rat brain atlas. ${ }^{18}$ The forebrain region was sectioned as a whole anterior to the hypothalamus.

\section{Stabilization of brain tissue}

Stabilization by conductive heat transfer was performed with the benchtop Stabilizor T1 instrument (Denator AB), according to the manufacturer's manual (www.denator .com). Whole brains were stabilized in "structure preserve mode," whereas smaller samples were stabilized in "autofresh sample mode." Samples were placed in a Maintainor Tissue card to ensure sufficient contact with the heat source, and the air was evacuated with a needle to reduce the risk of oxidation and ensure efficient heat transfer. Frozen samples were quickly moved from dry ice to prechilled cards, directly put in the Stabilizor, and then stored at $-80^{\circ} \mathrm{C}$ in the card.

\section{Tissue handling procedures (part I)}

The experimental design is depicted in Figure 1A-D. All test procedures were compared to our standard procedure (Fig. 1A), in which the brain is rapidly freed and, after

Table 1. Immunoreactive Peptide Levels in the Hypothalamus After Different Tissue Handling Procedures

\begin{tabular}{|c|c|c|c|c|}
\hline Tissue handling procedure & $D Y N B$ & $L A R G$ & $L A R G / D Y N B$ & $M E A P$ \\
\hline \multicolumn{5}{|l|}{ Nonstabilized brain } \\
\hline Standard procedure, freeze $(n=5)$ & $20.1 \pm 0.88$ & $44.5 \pm 1.83$ & $2.23 \pm 0.12$ & $73.4 \pm 2.18$ \\
\hline Wet ice 15 minutes, freeze $(n=4)$ & $18.8 \pm 0.65$ & $47.9 \pm 2.50$ & $2.55 \pm 0.09$ & $91.3 \pm 7.06$ \\
\hline Freeze, stabilize frozen before extraction $(n=6)$ & $17.8 \pm 1.48$ & $7.29 \pm 0.99 * *$ & $0.37 \pm 0.06^{* *}$ & $221 \pm 26.0 * *$ \\
\hline Stabilize, freeze $(n=4)$ & $35.1 \pm 2.14 *$ & $2.28 \pm 0.22 *$ & $0.07 \pm 0.01 *$ & $418 \pm 74.6^{*}$ \\
\hline \multicolumn{5}{|l|}{ Stabilized brain } \\
\hline Freeze $(n=4)$ & $36.6 \pm 1.05 *$ & $2.61 \pm 0.18^{*}$ & $0.07 \pm 0.01 *$ & $286 \pm 47.8 *$ \\
\hline Wet ice 15 minutes, freeze $(n=4)$ & $35.8 \pm 4.79 *$ & $3.25 \pm 0.11 *$ & $0.09 \pm 0.01 *$ & $263 \pm 15.9 *$ \\
\hline
\end{tabular}

Stabilization was tested at different time points according to Figure 1 and the peptide levels were compared with standard procedure (in bold) that is, the brain was rapidly freed and the hypothalamus removed and rapidly frozen on aluminum-encased dry ice. In addition, the consequences of keeping the tissue samples on wet ice for 15 minutes were tested in nonstabilized and stabilized brain.

The values represent mean \pm SEM and are expressed as fmol/mg tissue.

${ }^{*} p<0.05, * * p<0.01$, compared to standard procedure; ${ }^{\#} p<0.05$, compared to immediately frozen hypothalamus from stabilized brain. DYNB, dynorphin B; LARG, Leu-enkephalin-Arg ${ }^{6}$; MEAP, Met-enkephalin-Arg 6 -Phe ${ }^{7}$. 


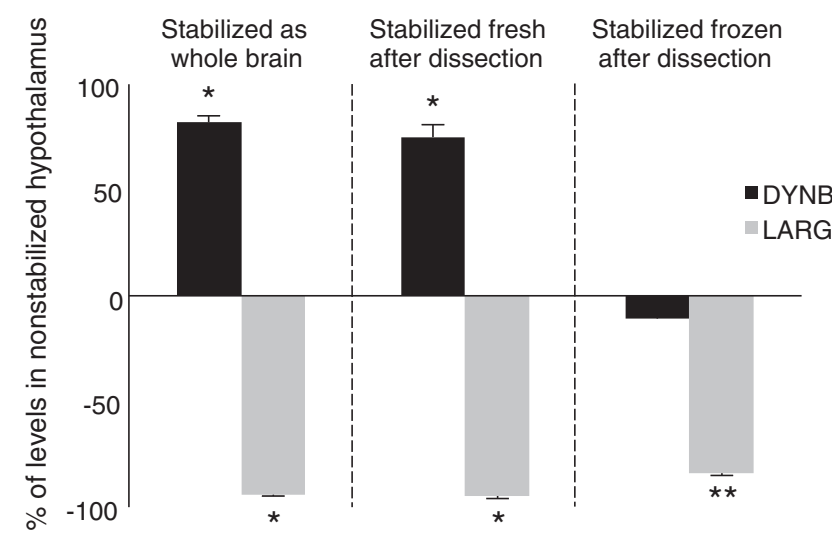

FIG. 3. Changes in prodynorphin-derived peptides in the hypothalamus after stabilization. Values are expressed as percent of immunoreactive (ir) levels in nonstabilized hypothalamus handled according to standard procedure. DYNB, dynorphin B; LARG, Leu-enkephalin-Arg ${ }^{6}{ }^{*} p<0.05$, $* * p<0.01$, compared to standard procedure.

hypothalamus removal, sectioned in a precooled matrix; regions of interest are dissected and rapidly frozen on aluminum-encased dry ice $\left(\approx-75^{\circ} \mathrm{C}\right)$. Test procedures were designed to answer the following questions:

(1) How does stabilization affect peptide levels? Critical time points for stabilization were tested for the following (Fig. 1B):

(a) Frozen hypothalamus immediately before extraction.

(b) Fresh hypothalamus, STR, or CCX immediately after removal or dissection.

(c) Whole brain before removal of hypothalamus or dissection.

(2) Do peptide levels change in samples kept on wet ice for 15 minutes? Peptide levels were measured in hypothalamus from nonstabilized and stabilized brain and in STR and CCX from nonstabilized brain (Fig. 1C). The choice of 15 minutes was based on the maximum time for a standard front-to-back dissection.

(3) How efficient is stabilization? Stabilized STR and CCX were kept at room temperature $\left(20^{\circ} \mathrm{C}\right)$ for 2 hours before freezing (Fig. 1D).

\section{Peptide extraction (part I)}

Samples were stored at $-80^{\circ} \mathrm{C}$ until extraction, which was done within 1 week. Homogenization and peptide extraction followed a standard procedure, ${ }^{8}$ that is, addition of $95^{\circ} \mathrm{C}$ acetic acid ( $1 \mathrm{M}, 1 \mathrm{~mL} / 50 \mathrm{mg}$ tissue $)$, heating $\left(95^{\circ} \mathrm{C}, 5 \mathrm{~min}-\right.$ utes), cooling on wet ice, homogenization $\left(4^{\circ} \mathrm{C}\right.$, Branson Sonifier $)$, reheating $\left(95^{\circ} \mathrm{C}, 5\right.$ minutes $)$, centrifugation $\left(4^{\circ} \mathrm{C}, 15\right.$ minutes, 12,000 g, Beckman GS-15R), and collection of the supernatant in Minisorb tubes. Extracts were further purified by ion-exchange chromatography (SP Sephadex C-25) and two fractions collected-fraction III (LARG and MEAP) and fraction V (DYNB). ${ }^{14}$ Samples were taken to dryness by vacuum centrifugation (Savant SpeedVac Plus SC210A) and stored $\left(-20^{\circ} \mathrm{C}\right)$ until analysis.

\section{Peptide extraction (part II)}

Stabilized hypothalamus and forebrain, respectively, were homogenized and pooled as a single sample before freezing. Aliquots thereof were subsequently subjected to five different extraction procedures (Fig. 2):

(1) Standard procedure, including seven steps as described above in part I.

(2) Acetic acid $\left(1 \mathrm{M}, 95^{\circ} \mathrm{C}\right)$ heated once $\left(95^{\circ} \mathrm{C}, 5\right.$ minutes), homogenization, and centrifugation.

(3) Acetic acid (1 M, room temperature), homogenization, and centrifugation.

(4) Weak acetic acid $(0.25 \%$, room temperature), homogenization, and centrifugation.

(5) Methanol/water/acetic acid (50/49.8/0.2, room temperature), homogenization, and centrifugation.

Samples were taken to dryness and stored as described above in Peptide extraction (part I).

\section{Peptide analysis}

The immunoreactive (ir) levels of DYNB, LARG, and MEAP were analyzed with well-established radioimmunoassays. ${ }^{8}$ Peptides, goat anti-rabbit IgG (GARGG), normal rabbit serum, and ${ }^{125}$ I DYNB were purchased from Bachem. All other chemicals were analytical grade and from common suppliers. For the DYNB antiserum $(113+, 1: 600,000)$, cross-reactivity was $100 \%$ with big dynorphin, $1 \%$ with DYNB(1-29), and none to other opioid peptides. For the

Table 2. Immunoreactive Peptide Levels in the Striatum After Different Tissue Handling Procedures

\begin{tabular}{|c|c|c|c|c|}
\hline Tissue handling procedure & $D Y N B$ & $L A R G$ & $L A R G / D Y N B$ & MEAP \\
\hline \multicolumn{5}{|l|}{ Nonstabilized brain } \\
\hline Standard procedure, freeze $(n=4)$ & $15.2 \pm 2.16$ & $33.7 \pm 3.05$ & $2.26 \pm 0.15$ & $132 \pm 5.79$ \\
\hline Wet ice 15 minutes, freeze $(n=4)$ & $16.9 \pm 4.61$ & $24.5 \pm 4.44$ & $1.57 \pm 0.22 *$ & $94.4 \pm 14.4^{*}$ \\
\hline Stabilize, freeze $(n=5)$ & $22.3 \pm 2.06$ & $3.66 \pm 0.28^{*}$ & $0.17 \pm 0.02 *$ & $333 \pm 34.5^{*}$ \\
\hline \multicolumn{5}{|l|}{ Stabilized brain } \\
\hline Freeze $(n=6)$ & $10.0 \pm 1.11$ & $3.29 \pm 0.43 * *$ & $0.35 \pm 0.06^{* *}$ & $32.7 \pm 6.30 * *$ \\
\hline Room temperature 2 hours, freeze $(n=6)$ & $13.3 \pm 2.04$ & $3.91 \pm 0.53 * *$ & $0.37 \pm 0.07 * *$ & $27.9 \pm 4.20 * *$ \\
\hline
\end{tabular}

Stabilization was tested at different time points according to Figure 1 and the peptide levels were compared with standard procedure (in bold) that is, the brain was rapidly freed and sectioned in a precooled matrix, regions of interest were dissected and rapidly frozen on aluminum-encased dry ice. In addition, the consequences of keeping the tissue samples on wet ice for 15 minutes was tested in nonstabilized brain and in room temperature $\left(20^{\circ} \mathrm{C}\right)$ for 2 hours in stabilized brain.

The values represent mean $\pm \mathrm{SEM}$ and are expressed as fmol/mg tissue.

${ }^{*} p<0.05,{ }^{*} p \leq 0.01$, compared to standard procedure. 


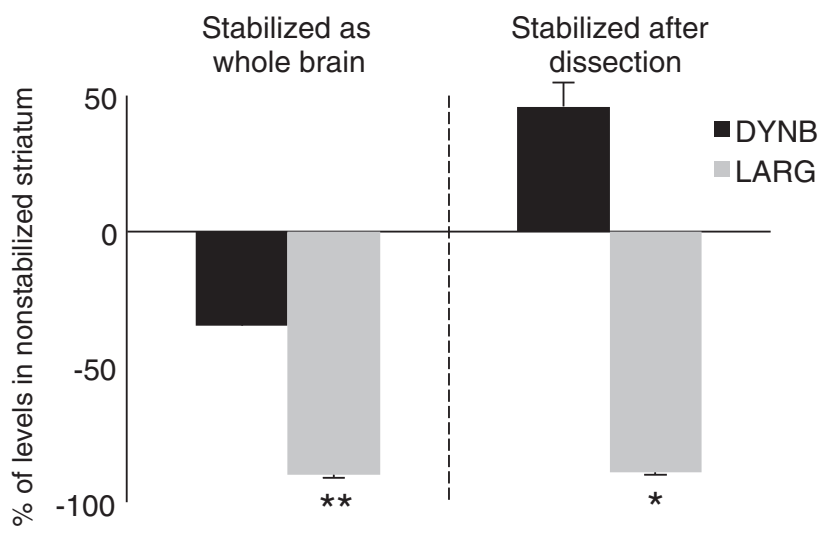

FIG. 4. Changes in prodynorphin-derived peptides in the striatum after stabilization. Values are expressed as percent of ir levels in nonstabilized striatum handled according to the standard procedure. $* p<0.05$, ** $p<0.01$, compared to standard procedure.

LARG antiserum (91:6D+, 1:60,000), cross-reactivity was $<0.01 \%$ for Leu-enkephalin and MEAP, $0.02 \%$ for DYNB, $0.04 \%$ for DYNA, and $0.08 \%$ for alpha-neoendorphin. The MEAP antiserum (90:3D(II), 1:160,000) cross-reactivity was $<0.1 \%$ for Met-enkephalin, Met-enkephalin-Arg ${ }^{6}$, Metenkephalin-Arg ${ }^{6}-\mathrm{Gly}^{7}-\mathrm{Leu}^{8}$, and Leu-enkephalin or LARG. All samples were run in triplicate.

\section{Statistical analyses}

The Kruskal-Wallis test was used for overall statistical analyses, and upon a statistical difference $(p<0.05)$, the Mann-Whitney $U$ test was used to confirm the significant difference $(p<0.05)$ between groups.

\section{Results}

\section{General observations}

The T1 log file revealed a height of $7.07 \pm 0.11 \mathrm{~mm}$ and stabilization time of $112.9 \pm 3.4$ seconds on brains of $1.82 \pm 0.02 \mathrm{~g}$ (mean \pm SEM) (Supplementary Fig. S1; Supplementary Data are available online at www.liebertpub .com/bio). The structure preserve program marginally compressed the brains, but dissection was uncomplicated; regions were still identifiable and easily separated.

The ex vivo time from decapitation until stabilization and/ or freezing is presented in Supplementary Table S1. The treatment time for stabilization of brains, fresh and frozen tissue was $107 \pm 3,21 \pm 1$, and $38 \pm 2$ seconds (mean \pm SEM), respectively. Temperature profiles for the postmortem handling groups are given in Supplementary Fig. S2.

\section{Hypothalamus}

The hypothalamus (Table 1) showed overall differences in ir levels of DYNB $(\mathrm{H}=20.2 ; p=0.001)$, LARG $(\mathrm{H}=24.3$; $p<0.001)$, and MEAP $(\mathrm{H}=20.5 ; p=0.001)$ levels. Since LARG can be generated enzymatically from DYNB, the LARG/DYNB ratio was calculated $(\mathrm{H}=22.4 ; p<0.001)$.

Effects of stabilization. DYNB increased in hypothalamus stabilized either alone or with the whole brain (Fig. 3). Stabilization of frozen hypothalamus immediately before extraction had no effect on DYNB levels, whereas LARG levels were generally reduced (Fig. 3). MEAP increased in all stabilized tissues compared to the standard procedure (Table 1). The LARG/DYNB ratio was lower in all stabilized tissue (Table 1).

Effects of 15 minutes on wet ice. There were no significant changes in ir DYNB, LARG, or MEAP levels, or the LARG/ DYNB ratio, in nonstabilized samples after 15 minutes on wet ice compared to the standard procedure (Table 1). Stabilized tissue kept on wet ice instead of being frozen after stabilization showed the same pattern, except for an increase in LARG.

\section{Striatum}

The STR (Table 2) showed overall differences in ir DYNB $(\mathrm{H}=10.3 ; p=0.035)$, LARG $(\mathrm{H}=16.7 ; p=0.002)$, MEAP ( $\mathrm{H}=20.7 ; p=0.0004)$ levels and the LARG/DYNB ratio $(\mathrm{H}=20.0 ; p=0.0005)$.

Effects of stabilization. The LARG levels, and consequently the LARG/DYNB ratio, were significantly lower in stabilized STR, whereas there was no significant difference in DYNB compared to the standard procedure (Fig. 4 and Table 2).

Table 3. Immunoreactive Peptide Levels in the Cingulate Cortex After Different Tissue Handling Procedures

\begin{tabular}{lccc}
\hline Tissue handling procedure & $D Y N B$ & $L A R G$ & $M E A P$ \\
\hline Nonstabilized brain & & & $\mathbf{2 . 7 0} \pm \mathbf{0 . 1 1}$ \\
$\quad$ Standard procedure, freeze $(\boldsymbol{n}=\mathbf{4})$ & $\mathbf{0 . 8 0} \pm \mathbf{0 . 1 1}$ & $3.61 \pm 0.84$ & $\mathbf{4 . 6 2} \pm \mathbf{0 . 5 2}$ \\
Wet ice 15 minutes, freeze $(n=4)$ & $1.07 \pm 0.12$ & ND & $4.95 \pm 1.49$ \\
Stabilize, freeze $(n=5)$ & $1.18 \pm 0.24$ & ND & $11.8 \pm 2.29 *$ \\
Stabilized brain & & ND & $2.76 \pm 0.50^{*}$ \\
Freeze $(n=6)$ & $0.93 \pm 0.08$ & $1.34 \pm 0.16$ & $3.19 \pm 1.02$ \\
$\quad$ Room temperature 2 hours $(n=6)$ & & & \\
\hline
\end{tabular}

Stabilization was tested at different time points according to Figure 1 and the peptide levels were compared with standard procedure (in bold) that is, the brain was rapidly freed and sectioned in a precooled matrix, regions of interest were dissected and rapidly frozen on aluminum-encased dry ice. In addition, the consequences of keeping the tissue samples on wet ice for 15 minutes were tested in nonstabilized brain and in room temperature $\left(20^{\circ} \mathrm{C}\right)$ for 2 hours in stabilized brain.

The values represent mean \pm SEM and are expressed as fmol/mg tissue.

${ }^{*} p<0.05$, compared to standard procedure.

$\mathrm{ND}$, not detectable in the radioimmunoassay. 
Table 4. Immunoreactive Peptide Levels in Stabilized Tissue After Different Extraction Procedures

\begin{tabular}{|c|c|c|c|c|}
\hline \multicolumn{2}{|c|}{ Test procedure } & $D Y N B$ & $L A R G$ & $M E A P$ \\
\hline \multicolumn{5}{|c|}{ A. Hypothalamus } \\
\hline 1 & $1 \mathrm{M}$ acetic acid, $2 \times 95^{\circ} \mathrm{C}$ & $34.8 \pm 3.92$ & ND & $433 \pm 21.4$ \\
\hline 2 & $1 \mathrm{M}$ acetic acid, $1 \times 95^{\circ} \mathrm{C}$ & $34.0 \pm 3.34$ & ND & $386 \pm 20.3$ \\
\hline 3 & $1 \mathrm{M}$ acetic acid & $42.1 \pm 2.21$ & ND & $436 \pm 22.9$ \\
\hline 4 & $0.25 \%$ acetic acid & $34.5 \pm 2.14$ & ND & $471 \pm 16.1$ \\
\hline 5 & Methanol/water/acetic acid & $33.1 \pm 1.57$ & ND & $489 \pm 34.0$ \\
\hline \multicolumn{5}{|c|}{ B. Forebrain } \\
\hline 1 & $1 \mathrm{M}$ acetic acid, $2 \times 95^{\circ} \mathrm{C}$ & $11.8 \pm 0.34$ & $1.70 \pm 0.04$ & $128 \pm 2.41$ \\
\hline 2 & $1 \mathrm{M}$ acetic acid, $1 \times 95^{\circ} \mathrm{C}$ & $10.9 \pm 0.29$ & $1.40 \pm 0.08 *$ & $131 \pm 4.84$ \\
\hline 3 & $1 \mathrm{M}$ acetic acid & $12.0 \pm 0.32$ & $1.37 \pm 0.05 * *$ & $108 \pm 2.60 * *$ \\
\hline 4 & $0.25 \%$ acetic acid & $9.78 \pm 0.20 * *,+++$ & $1.18 \pm 0.04 * * *,+$ & $111 \pm 2.76 * *$ \\
\hline 5 & Methanol/water/acetic acid & $9.21 \pm 0.28 * * *,+++$ & $1.19 \pm 0.07 * * *$ & $129 \pm 7.24$ \\
\hline
\end{tabular}

The ir peptide levels were measured in pooled stabilized hypothalamus (A) and forebrain (B), respectively, after different extraction procedures according to Figure 2.

$* p<0.05 ; * * p<0.01 ; * * * p<0.001$ compared to test procedure 1 (standard procedure).

${ }^{+} p<0.05 ;{ }^{++} p<0.001$ compared to test procedure 3 .

ir, immunoreactive.

MEAP levels were lower in STR from whole stabilized brain and higher in STR stabilized immediately after dissection from nonstabilized brain (Table 2).

Effects of 15 minutes on wet ice. There were no significant changes in ir DYNB or LARG, but MEAP levels and the LARG/DYNB ratio decreased in nonstabilized STR samples after 15 minutes on wet ice (Table 2).

Efficacy of stabilization. Levels of DYNB, LARG, MEAP or the DYNB/LARG ratio were unaffected by leaving stabilized STR at room temperature for 2 hours compared to immediately frozen stabilized samples (Table 2).

\section{Cingulate cortex}

The CCX (Table 3) showed an overall difference of ir $\operatorname{MEAP}(\mathrm{H}=13.0 ; p=0.012)$, but not DYNB levels $(\mathrm{H}=6.73$; $p=0.151)$. LARG levels were below detection in all stabilized tissue.

Effects of stabilization. In CCX, the LARG levels were reduced below detection limit in all stabilized tissues, whereas DYNB levels were unchanged (Table 3). Higher levels of MEAP were detected in samples immediately stabilized and lower in samples from stabilized whole brains, compared to the standard procedure.

Effects of 15 minutes on wet ice. No significant changes were observed in the CCX for DYNB, LARG, or MEAP levels after leaving nonstabilized samples on wet ice for 15 minutes (Table 3).

Efficacy of stabilization. Levels of DYNB or MEAP (LARG below detection) were unaffected by leaving stabilized CCX at room temperature for 2 hours compared to immediately frozen stabilized samples (Table 3).

\section{Extraction protocols for stabilized samples}

Analysis showed no overall differences between extraction protocols for ir DYNB or MEAP levels in stabilized hypothalamus, and LARG levels were below the limit of detection (Table 4A). In the forebrain, overall differences were detected in DYNB $(\mathrm{H}=27.4 ; p<0.001)$, LARG $(\mathrm{H}=22.4 ; p<0.001)$, and MEAP $(\mathrm{H}=16.3 ; p<0.01)$ levels. Table 4B shows that procedures with the weaker acetic acid
(4) or a methanol/water/weak acetic acid (5) extraction media reduced levels of DYNB compared to the standard procedure with heating cycles and stronger acetic acid (1 and 3). All procedures with reduced heating (2-3) and weaker acetic acid or methanol/water extraction media (4-5) led to reduced levels of LARG, whereas levels of MEAP were reduced after procedures without heating cycles (3) and weaker acetic acid (4).

\section{Discussion}

\section{Methodological considerations}

Keeping nonstabilized samples on wet ice for 15 minutes instead of immediately freezing them had no impact on DYNB or LARG in the hypothalamus, STR, or CCX, indicating this as a noncritical step for these peptides. However, region-specific changes were noted for MEAP, which calls for caution; MEAP was unaffected in hypothalamus and CCX, but reduced in STR.

Stabilization was tested at four critical time points: whole brain stabilized immediately upon removal from the skull, regions of interest stabilized promptly upon dissection, hypothalamus immediately frozen and stabilized directly from the frozen state, and stabilized samples kept for 2 hours at room temperature before freezing.

Stabilized hypothalamus had higher DYNB and lower LARG levels. LARG is generated through enzymatic cleavage of dynorphins, ${ }^{1,19}$ and it is therefore assumed that heat-inactivated enzymes and delayed postmortem turnover resulted in increased substrate levels and decreased product. These findings are in line with a study using in vivo microwave irradiation to stabilize brain tissue. ${ }^{8}$ In STR and CCX the impact of stabilization on DYNB was not statistically significant, but the effects on LARG mirrored those in the hypothalamus, that is, markedly reduced levels upon stabilization. These results highlight regional differences and indicate that both the enzymatic generation of LARG and the production of DYNB from precursors were potentially impeded by stabilization.

Stabilization of frozen hypothalamus before extraction reduced LARG levels compared to standard procedure, but 
not to the same extent as stabilization of fresh tissue, whereas stabilization of frozen tissue had no impact on DYNB. These results imply that distinct processing steps are active during stabilization of fresh and frozen samples, and that the limited time of temperature rise-either through addition of hot acetic acid or during stabilization-is sufficient to impose a critical step, especially for DYNB.

Analysis of MEAP revealed markedly higher levels in the hypothalamus after stabilization of either fresh or frozen tissue, indicating major postmortem metabolism during standard procedures. These changes apparently occurred immediately postmortem as the levels in nonstabilized hypothalamus on wet ice 15 minutes were unchanged. A different pattern was evident in STR and CCX in which the timing of stabilization appeared to be critical. When these were dissected from nonstabilized brain and then stabilized, MEAP levels were higher. In contrast, stabilization of whole brain before dissection resulted in lower levels, that is, the opposite effect compared to hypothalamus.

Thus, the results revealed region-specific effects of stabilization; these were more pronounced for MEAP. This is not surprising considering the tissue-specific processing of endogenous opioids. ${ }^{19,20}$ Stabilization affects all enzymatic processes, both degradation and processing from precursors. Furthermore, our findings underline the high efficacy of stabilization and imply that rapid freezing after sample collection can be omitted, greatly simplifying the experiment. Ultimately, even with exposure to room temperature for 2 hours, the peptide levels remained unchanged in stabilized tissues.

\section{Comparisons with microwave irradiation}

The major advantage with microwave irradiation is that protein inactivation and decapitation are simultaneous, but it risks introducing architectural artifacts from formation of hotspots with temperatures above the boiling point. Stabilization runs an inevitable small risk of metabolism during the limited ex vivo time, but advantages include ease of use, versatility, and-as treatment time is adjusted to sample size-it avoids artifacts by controlled heating (entire sample $>90^{\circ} \mathrm{C}$ and $<95^{\circ} \mathrm{C}$ ).

\section{Extraction protocols for stabilized tissue}

Stabilization eliminated the need for heating during extraction as DYNB levels were unchanged and reduced for LARG and MEAP, indicating the loss of peptide during heating. A reduced concentration of acetic acid or use of methanol/water/acetic acid reduced DYNB and LARG, but not MEAP; peptide hydrophilicity was a key factor in optimizing the extraction media.

\section{Conclusion}

In conclusion, the results reveal markedly reduced postmortem changes in ir opioid peptide levels in stabilized tissue compared to standard procedures. The identification of region- and peptide-specific critical steps will minimize postmortem alterations in tissue samples, optimize peptide analysis, and enable more correct functional interpretations. The conductive heat transfer method described herein offers an easy-to-use and efficient benchtop technique for optimizing peptide analysis. Stabilization reduces the need for rapid freezing and facilitates handling of biological samples from sampling to storage, which is especially important in a clinical setting. Loss of, or alterations in, biomarkers or introduction of artifacts can be avoided and the diagnostic and research value of the sample will be preserved.

\section{Acknowledgments}

Financial support from the Swedish Research Council (K2012-61X-22090-01-3) and the Alcohol Research Council of the Swedish Alcohol Retail Monopoly (20140040) are gratefully acknowledged. The authors are grateful to Dr. Mats Borén (Denator AB) for practical instructions and Dr. Sara Palm for preparation of figures.

\section{Author Disclosure Statement}

No conflicting financial interests exist.

\section{References}

1. Hallberg M, Nyberg F. Neuropeptide conversion to bioactive fragments-an important pathway in neuromodulation. Curr Protein Pept Sci 2003;4:31-44.

2. Hallberg M. Neuropeptides: Metabolism to bioactive fragments and the pharmacology of their receptors. Med Res Rev 2015;35:464-519.

3. Scopes RK. Protein Purification: Principles and Practice, 3rd ed. New York: Springer-Verlag; 1994.

4. Svensson M, Borén M, Sköld K, et al. Heat stabilization of the tissue proteome: A new technology for improved proteomics. J Proteome Res 2009;8:974-981.

5. Kultima K, Sköld K, Borén M. Biomarkers of disease and post mortem changes-heat stabilization, a necessary tool for measurement of protein regulation. J Proteomics 2011; 75:145-159.

6. Lenox RH, Gandhi OP, Meyerhoff JL. Microwave applicator for in vivo rapid inactivation of enzymes in the central nervous system. IEEE Trans Microw Theory Tech 1976;24:58-61.

7. Mathé A, Stenfors C, Brodin E, et al. Neuropeptides in brain: Effects of microwave irradiation and decapitation. Life Sci 1990;46:287-293.

8. Nylander I, Stenfors C, Tan-No K, et al. A comparison between microwave irradiation and decapitation: Basal levels of dynorphin and enkephalin and the effect of chronic morphine treatment on dynorphin peptides. Neuropeptides 1997;31:357-385.

9. Theodorsson E, Stenfors C, Mathé A. Microwave irradiation increases recovery of neuropeptides from brain tissues. Peptides 1990;11:1191-1197.

10. Comb M, Seeburg PH, Adelman J, et al. Primary structure of the human Met- and Leu-enkephalin precursor and its mRNA. Nature 1982;295:663-666.

11. Nakanishi S, Inoue A, Kita T, et al. Nucleotide sequence of cloned cDNA for bovine corticotropin-beta-lipotropin precursor. Nature 1979;278:423-427.

12. Kakidani H, Furutani Y, Takahashi H, et al. Cloning and sequence analysis of cDNA for porcine beta-neoendorphin/dynorphin precursor. Nature 1982;298:245-249.

13. Akil H, Owens C, Gutstein H, et al. Endogenous opioids: Overview and current issues. Drug Alcohol Depend 1998; 51:127-140.

14. Spanagel R, Herz A, Shippenberg TS. Opposing tonically active endogenous opioid systems modulate the mesolimbic dopaminergic pathway. Proc Natl Acad Sci U S A 1992; 89:2046-2050. 
15. Steiner $\mathrm{H}$, Gerfen CR. Role of dynorphin and enkephalin in the regulation of striatal output pathways and behavior. Exp Brain Res 1998;123:60-76.

16. Svensson M, Sköld K, Nilsson A, et al. Neuropeptidomics: Expanding proteomics downwards. Biochem Soc Trans 2007; 35:588-593.

17. Rountree BC, Van Kirk CA, You H, et al. Clinical application for the preservation of phosphoproteins through in situ tissue stabilization. Proteome Sci 2010;8:61.

18. Paxinos G, Watson C. The Rat Brain in Stereotaxic Coordinates, 6th ed.San Diego, CA: Academic Press; 2009.

19. Berman Y, Rattan AK, Carr K, et al. Regional distribution of neuropeptide processing endopeptidases in adult rat brain. Biochimie 1994;76:246-250.
20. Zamir N, Weber E, Palkovits M, et al. Differential processing of prodynorphin and proenkephalin in specific regions of the brain. Proc Natl Acad Sci U S A 1984;81:6886-6889.

Address correspondence to: Ingrid Nylander, PhD Department of Pharmaceutical Biosciences, Neuropharmacology, Addiction \& Behavior Uppsala University Box 591 SE-75124 Uppsala Sweden

E-mail: ingrid.nylander@farmbio.uu.se 